\title{
TEMPORAL CHANGES IN SEMIVARIOGRAM OF OCEAN SURFACE LATENT HEAT FLUX UNDER LINEAR TREND
}

\author{
M. K. Singh*, P. Venkatachalam
}

CSRE, IIT Bombay, Powai, Mumbai, 400076 (manojks,pvenk)@iitb.ac.in

KEY WORDS: Temporal, Spatial, Statistics, Oceanography, Prediction

\begin{abstract}
:
One of the ways to study spatio-temporal variability of a process is to consider it as a temporal variation of a spatial process. Semivariogram is a measure of spatial variation in a process. If a process is undergoing a linear trend, then semivariogram parameters such as range, sill and nugget are bound to change. In this paper, a mathematical closed form of range, sill, and nugget and in turn semivariogram were expressed for a process under linear trend. The derived semivariogram was used to study the latent heat flux (LHF) over the Indian Ocean. LHF values depend on sea surface temperature (SST) and wind speed (WS) over ocean surface. Universal kriging (UK) was used to estimate the LHF with WS and SST as covariables. UK coefficients corresponding to covariables were found out for the years 2010, 2020, 2030, 2040 and 2050. In similar line, study has been attempted to see how empirical orthogonal function modes of a spatio-temporal process change with time under linear trend.
\end{abstract}

\section{INTRODUCTION}

There are several ways to interpret a spatio-temporal process. The process can be thought as a spatial process which evolves with time. Study of the spatial behavior of the process with time is important in many fields. As an example, temporal behavior of LHF with respect to changes in WS and SST was considered in this paper. Study of LHF is important as it is a key component of hydrological cycle. In recent decades signification positive trend in LHF of World Ocean was observed. WS and SST values have also increased in recent times. Therefore study of LHF along with WS and SST is interesting ( $\mathrm{Li}$ et al., 2011). Universal kriging method was used in this study, to relate LHF with their covariables. Kriging coefficients and their variances can be computed using equations UK systems of equation. The UK coefficients determine the contribution of WS and SST on LHF. The variance of the parameters determine their reliability. It will be interesting to see how these coefficients change and their variances change with time. Therefore, the problem statements are

1. Let $\beta_{0}, V\left(\beta_{0}\right), \beta_{k}$ and $V\left(\beta_{k}\right)$ represent the UK coefficient vector and the variance matrix at time $t=0$ and at time $t=$ $k$ respectively. Then given $\beta_{0}$ and $V\left(\beta_{0}\right)$ can we estimate $\beta_{k}$ and $V\left(\beta_{k}\right)$ ?

2. If we know the semivariogram $\gamma_{0}$ at time $t=0$, can we estimate semivariogram $\gamma_{k}$ at time $k$

This work was centered towards studying these problems under assumption that the process and its covariates have linear trend. Temporal behavior of semivariogram is discussed in next section and the results related to temporal changes in UK coefficients and their variances are discussed in section 3.. More about universal kriging can be found in (Chiles and Delfiner, 2009)

\section{METHODOLOGY}

At time $t=0$, the $\operatorname{LHF} Y_{0}(s)$ is given by

$$
Y_{0}(s)=\mu(s)
$$

\footnotetext{
${ }^{*}$ Corresponding author.
}

If we assume LHF has annual trend $\omega_{l}$, then, after $k$ years, LHF will be,

$$
Y_{k}(s)=\mu(s)+\omega_{l}(s) \times k
$$

Trends can also be considered as a spatial process. Let us denote $\gamma(h)$ as the semivariogram of LHF trend. Then,

$$
\begin{aligned}
\gamma_{k}(h) & =\frac{1}{2 N} \sum_{s}\left[y_{k}(s+h)-y(s)\right]^{2} \\
& =\frac{1}{2 N} \sum_{s}\left[\mu(s+h)+\omega_{l}(s+h) \times k-\mu(s)-\omega_{l}(s) \times k\right]^{2} \\
& =\frac{1}{2 N} \sum_{s}\left\{[\mu(s+h)-\mu(s)]^{2}+k^{2}\left[\omega_{l}(s+h)-\omega_{l}(s)\right]^{2}\right\} \\
& =\gamma_{0}(h)+k^{2} \gamma(h)
\end{aligned}
$$

the non-square term not shown in equation 5 is sum of equal but opposite numbers and hence zero. In equation $6, \gamma_{k}(h)$ increases as $k$ increases. Since $\gamma_{0}(h)>>\gamma(h)$, for small values of $k$, spatial variability does not change significantly. As time changes significantly i.e. $k$ is large enough, $k^{2} \gamma(h)$ cannot be neglected from $\gamma(h)$. For the purpose of this study we assume that semivariogram is a continuous function of $h$, with a possible exception at $h=0$. For $h$ greater than a distance, called as range, semivariogram is constant. From equation 5, it is easy to conclude that, the range of $\gamma_{k}(h)$ will be maximum of the range of $\gamma_{0}(h)$ and $\gamma(h)$. Sill $S_{k}$ of $\gamma_{k}(h)$ will be given by,

$$
S_{k}=S_{0}+k^{2} \times S
$$

where $S_{0}$ is sill of $\gamma_{0}$ and $S$ is sill of $\gamma$. Once range and sill are known, closed form of semivariogram can be found.

Kriging system of equation can be solved using variance covariance matrix, instead of semivariogram. Properties of variance similar to that in the equation 6 , can also be developed. If $\gamma$ and covariance function $C$ are continuous function of $h$, then

$$
\begin{aligned}
\lim _{h \rightarrow \infty} \gamma(h) & =C(0)-\lim _{h \rightarrow \infty} C(h) \\
\lim _{h \rightarrow \infty} \gamma(h) & =C(0)
\end{aligned}
$$

In practice we assume $\gamma(h)$ to be constant for $h$ greater than range. Therefore, variance $C(0)$ can be written as semivariogram of large $h$. Let $C_{0}(h)$ and $C_{k}(h)$ be variance at time $t=0$ and 
time $k=0$. If the process is second order stationary, then

$$
\begin{aligned}
C_{k}(h) & =C_{k}(0)-\gamma_{k}(h) \\
& =\gamma_{k}(R)-\gamma_{k}(h) \\
& =\gamma_{0}(R)+k^{2} \gamma(R)-\gamma_{0}(h)+k^{2} \gamma(h) \\
& =\gamma_{0}(R)-\gamma_{0}(h)+k^{2}(\gamma(R)-\gamma(h)) \\
& =C_{0}(0)-\gamma_{0}(h)+k^{2}[C(0)-\gamma(h)] \\
& =C_{0}(h)-k^{2} C(h)
\end{aligned}
$$

where $R$ is the maximum of the ranges of $\gamma(h)$ and $\gamma_{0}(h)$. The covariance matrix at time $t$ can be written as

$$
\Sigma_{\hat{\beta}}^{(k)}=\left(B_{n}^{(k) T} V_{(k)}^{-1} B_{n}^{(k)}\right)^{-1}
$$

and calculated coefficient vector $\hat{\beta}$ will be given as,

$$
\hat{\beta}_{(k)}=\Sigma_{\hat{\beta}}^{(k)} B_{n}^{(k) T} V_{(k)}^{-1} Z_{n}^{(k)}
$$

where $B_{n}$ is a matrix of size $n \times 3$, first column of which is filled with 1 , second column represents wind speed trend and third column represents SST trend for $n$ locations. $Z_{n}^{(k)}$ represents true LHF at time $k$.

To establish a relationship between $\Sigma_{\hat{\beta}}^{(k)}$ and $\Sigma_{\hat{\beta}}^{(r)}$ and between $\hat{\beta}_{(k)}$ and $\hat{\beta}_{(r)}$, we need to know relationship between $B_{n}^{(k)}$ and $B_{n}^{(k)}$ and between $Z_{n}^{(k)}$ and $Z_{n}^{(r)}$. It is not an easy task to establish such relationships. Nevertheless, we can still be able to find $\Sigma_{\hat{\beta}}^{(k)}$ and $\hat{\beta}_{(k)}$ by direct calculations. The results related to direct computation of universal kriging of LHF with WS and SST as co variables are summarized in the next section.

\section{RESULTS AND DISCUSSION}

Table 1 shows the UK coefficients, their variance and covariance.

\begin{tabular}{lccccc}
\hline & 2010 & 2020 & 2030 & 2040 & 2050 \\
\hline Const & 0.064 & 0.121 & 0.179 & 0.23 & 0.29 \\
WS & 17.30 & 17.36 & 17.38 & 17.392 & 17.398 \\
SST & 18.83 & 18.89 & 18.91 & 18.930 & 18.937 \\
V(Const) & 4.67 & 8.64 & 14.02 & 20.83 & 29.05 \\
V(WS) & 1.32 & 0.71 & 0.54 & 0.46 & 0.42 \\
V(SST) & 6.00 & 3.22 & 2.45 & 2.10 & 1.91 \\
Cov(Const,WS) & -0.083 & -0.083 & -0.092 & -0.10 & -0.118 \\
Cov(Const,SST) & -0.616 & -0.614 & -0.683 & -0.77 & -0.86 \\
Cov(WS,SST) & -0.242 & -0.13 & -0.098 & -0.084 & -0.076 \\
\hline
\end{tabular}

Table 1: UK coefficients and their variability expected in coming decades

Following observations can be interpreted from the table:

1. Contribution from parameters other than WS and SST increases with time, although the contribution is small as compared to WS and SST. The variance of factors other than WS and SST increases very fast and therefore their reliability decreases with time.

2. WS and SST coefficients increase at a very small rate but their variances decrease very quickly. Therefore, their reliability increases with time. In most of the regions LHF, WS and SST have positive trend. Therefore, it is expected to find increment in the UK coefficients.
3. The three variables are negatively correlated. Correlation between WS and SST with other parameters increases with time but between WS and SST decreases.

\section{ACKNOWLEDGEMENTS}

The authors thank INCOIS, Hyderabad team for providing the data set of latent heat flux, wind speed and sea surface temperature

\section{REFERENCES}

Chiles, J.-P. and Delfiner, P., 2009. Geostatistics: modeling spatial uncertainty. Vol. 497, John Wiley \& Sons.

Li, G., Ren, B., Yang, C. and Zheng, J., 2011. Revisiting the trend of the tropical and subtropical pacific surface latent heat flux during 1977-2006. Journal of Geophysical Research 116(D10), pp. D10115. 